\title{
Evolutionary Study of the Retinal Nerve Fiber Layer for the Patients with Glaucoma and Diabetes Mellitus
}

\author{
O. I. Motas ${ }^{1}$, D. Chiselita ${ }^{1}$, Nicoleta Anton Apreutesei ${ }^{1}$, Vesna K. Hristova ${ }^{2}$, \\ Teresa Bonacci ${ }^{3}$, M. Ayaz Ahmad ${ }^{4}$ \\ ${ }^{1}$ Department of Ophthalmology, Faculty of Medicine, University of Medicine, “Grigore T. Popa” Ias, Romania \\ ${ }^{2}$ Faculty of Biotechnical Sciences, University of St. Clement Ohridski, Partizanska, bb 7000 Bitola, Macedonia \\ ${ }^{3}$ Dipartimento di Biologia, Ecologia e Science Delta Terra, Universita Delta Calabria, Via Pietro Bucci, 87036 \\ Rende, CS, Italy \\ ${ }^{4}$ Physics Department, Faculty of Science, P.O. Box 741, University of Tabuk - 71491, Saudi Arabia.
}

\begin{abstract}
The aim of the paper was the evaluation of the modifications of the retinal nerve fiber layer for the patients with primary open angle glaucoma or ocular hypertension associated with diabetes mellitus. Material and method: a prospective study that included a number of 25 patients. The study group was made up of 17 patients: 12 patients with primary open angle glaucoma (POAG) and diabetes mellitus and 5 patients with ocular hypertension $(\mathrm{OH})$ and diabetes mellitus. The control group included 8 patients without glaucoma. All the patients with an accurate diagnosis of primary open angle glaucoma and intraocular hypertension that were medically and/or surgically treated or not, associated with diabetes mellitus, were included in the study. The patients with other types of glaucoma (closed angle, pigmentary, cortisone related, pseudoexfoliative), the ones without an accurate diagnosis of glaucoma or without associated diabetes mellitus were excluded. The control group was made up of subjects without a glaucoma diagnosis or clinical elements related to glaucoma, without surgical interventions in their medical records. All participants were evaluated with CIRRUS HD-OCT.
\end{abstract}

Keywords - Poag, Oh, Rnfl, Cirrus Hd-Oct, Diabetes Mellitus.

\section{INTRODUCTION}

Diabetes and glaucoma were two chronic diseases that greatly affected the population over 40 years old. The two diseases had a degenerative effect on the nervous structures of the eyeball, as well as on the visual pathway. Glaucoma was a chronic neuropathy which determined the progressive death of the retinal ganglion cells and their axons [1]. Those structural modifications could be perimetrically identified when at least $30 \%$ of the retinal ganglion cells were destroyed. The identification, documenting and analysis in due time of those modifications would allow an early diagnosis before the significant impairment of the visual function [2]. The OCT (optical coherence tomography) could identify the modifications on the level of the optic nerve and the retinal nerve fibers before the impairment of the visual function. Huang used it for the first time in 1991 and it had a very rapid evolution and thus the current models were highly performant [3]. They applied the laser interferometry principle in order to achieve in vivo acquisition of images representing sections of the retinal structures, at a resolution of about $10 \mu \mathrm{m}$ (classic model). The recent models (Spectral Domain OCT) had an axial resolution of 5-6 microns and the scanning speed was very high (55000 scans/second), thus increasing the precision of the examination and improving the visualization of the retinal nerve fiber layer [2, 4]. The device had scanning protocols for the optical nerve papilla imaging, respectively the retinal nerve fiber layer, the measurement of the macular layers, useful morphological information for the diagnosis and the management of the patients with diabetic macular edema. The quantitative parameters mostly used in the present were: diameters (of the optic disc and the excavation), surfaces, reports (C/D). The circumpapillary scans measured the thickness of the retinal nerve fiber layer (as shown in figure 1). The resulted profiles were compared against normative bases: when the curve of the nervous layer was located on the green line was considered to be normal, on the yellow line must be interpreted as suspicious and on the red line must be labeled as abnormal.

The most important quantitative parameters were the total thickness, the thickness in the upper and lower sectors. Several studies showed that the measurement of the thickness of the retinal nerve fiber layer was an important parameter in the early diagnosis of glaucoma $[5,6]$. 


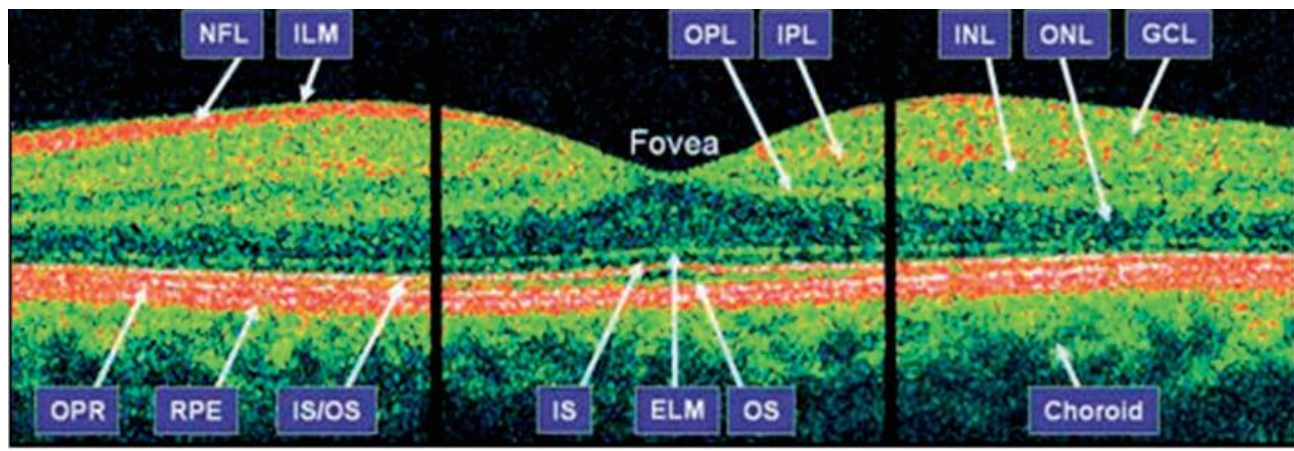

Fig. 1. Cirrus HD-OCT B-scan. GCL - ganglion cell layer; IPL - inner plexiform layer; OPL - outer plexiform layer; RPE - retinal pigment epithelium; IS - inner photoreceptor segments; IS/OS - IS/OS junction; OPR - outer photoreceptors; OS - outer photoreceptor segments; PRL = photoreceptor layer; RPE = retinal pigment epithelium; ELM = external limiting membrane; NFL - nerve fiber layer; ILM - inner limiting membrane; INL inner nuclear layer; ONL - outer nuclear layer.

\section{MATERIALS AND METHOD USED}

A comparative study that included a number of 25 patients, 12 patients with primary open angle glaucoma (POAG) and diabetes mellitus and 5 patients with ocular hypertension $(\mathrm{OH})$ and diabetes mellitus (study group) and the rest of 8 patients without glaucoma (normal) that made up the control group. The study was conducted for a period of 18 months including patients from the $\mathrm{I}^{\text {st }}$ Ophthalmology Clinic within "Sf. Spiridon" Hospital, Iasi. The inclusion criteria were: normal and hypertensive primary open angle glaucoma, intraocular hypertension and associated diabetes. The patients with other types of glaucoma were excluded (pseudoexfoliative, pigmentary, cortisone related, neovascular and primary closed angle) and those without associated diabetes. All patients underwent a complete eye exam that also included the determination of the best corrected visual acuity and the following parameters for glaucoma: determination of the intraocular pressure (with aplanotonometer Goldmann), perimetral evolution (through automated perimetry with Humphrey Field Analyzer II), the fundus of the eye evaluated with Volk 78D lens (absence, presence and progression RD) and the OCT exam (evaluation of the optic disc and the retinal nerve fiber layer). The age of the patients, the visual acuity level and the examined eye were recorded. All participants were questioned regarding ocular surgical interventions and acute retinal affections.

The criteria for normal and hypertensive POAG diagnosis were: over 35 years old, normal or $>21 \mathrm{~mm} \mathrm{Hg}$ IOP without treatment, open camerular angle following gonioscopy, impairment of the optic nerve specific to glaucoma (ratio C/D > 0.5), abnormal visual field (through perimetry Humphrey Field Analyzer) and the retinal nerve fiber layer with diffuse or localized defects (optic coherence tomography - Cirrus HD OCT Zeiss) [1]. The intraocular hypertension diagnosis according to the glaucoma guide was based on the followings: age over 35 years old, IOP over $21 \mathrm{mmHg}$, visual field and normal aspect of the optic nerve [1]. All

participants were included in the examination with Cirrus HD-OCT, with a single operator, applying the scanning protocol Optic Disc Cube 200×200, in order to measure the thickness of the retinal nerve fiber layer. One of the three successive scans, the one with the maximum signal power, was included in our study. The average thickness of the RNFL was analyzed, as well as the thickness of the layers in the four sectors (upper, lower, nasal and temporal). $\quad$ For the statistical analysis of the results, the Sigma Plot (version 11) medical statistical program was used. In the test $t$ student the values of $p<0.05$ were considered statistically significant. The data was reported as average \pm standard deviation.

\section{RESULTS AND DICUSSIONS}

The study included 12 patients (48\%) ( 9 women and 3 men) with primary open angle glaucoma and diabetes, 5 patients (20\%) (4 women and one man) with intraocular hypertension and diabetes mellitus and 8 healthy subjects (32\%) (as it is depicted in figure 2). The median age was $64.29 \pm 5.3$ years in the glaucoma and diabetes group, $60.5 \pm 5.45$ years in the ocular hypertension and diabetes group and $62.2 \pm 4.76$ years in the control group. Table 1 and 2 described the clinical features of the patients from the study groups. There were no statistically significant differences between the three groups regarding the median age. The average MD in the group with glaucoma and ocular hypertension was $-2.39 \pm 0.10$, respectively $1.81 \pm 0.88$. The average PSD in the group with glaucoma and ocular hypertension was $-0.8 \pm 0.62$, respectively $1.81 \pm 0.88$ (Table I and II). There were statistically significant differences and negative correlation in the group with glaucoma and ocular hypertension regarding the MD, PSD and the thickness of the RNFL. $(p<0.001$ in the glaucoma group and $p=$ 0.008 in the ocular hypertension group). 


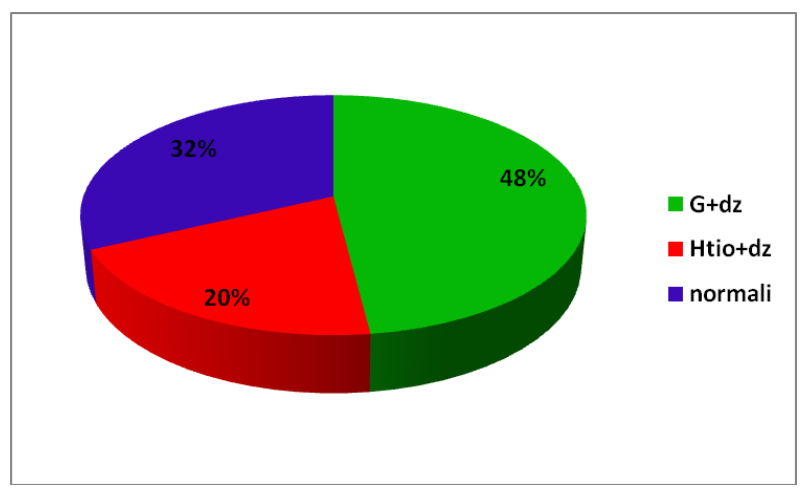

Fig. 2. Distribution of the patients according to study groups. G+DM (primary open angle glaucoma and diabetes mellitus), $\mathrm{OH}+\mathrm{DM}$ (intraocular hypertension and diabetes mellitus).

Table I: Patients with glaucoma and diabetes mellitus (group 1), clinical characteristics

\begin{tabular}{|l|c|c|c|c|c|c|c|}
\hline Patient & Diagnostic & Age & VA & Eye & C/D & MD & PSD \\
\hline 1. C.C. & POAG, DM & 67 & 1 & OD & 0.57 & -0.95 & 1.92 \\
\hline 2. P.C. & POAG, DM & 59 & 0.6 & OD & 0.7 & -0.15 & 1.55 \\
\hline 3. H.V. & POAG, DM & 73 & 0.7 & OD & 0.71 & -0.6 & 1.79 \\
\hline 4. C.E. & POAG, DM & 70 & 1 & OD & 0.57 & 0.05 & 0.5 \\
\hline 5. A.L. & POAG, DM & 61 & 1 & OD & 0.55 & 0.6 & 1.2 \\
\hline 6. P.E. & NTG, DM & 70 & 1 & OD & 0.73 & -5.37 & 5.47 \\
\hline 7. P.P. & NTG, DM & 59 & 1 & OD & 0.68 & -3.20 & 1.30 \\
\hline 8. A.E. & POAG, DM & 67 & 0.8 & OS & 0.58 & -4.8 & 7.89 \\
\hline 9. G.V. & NTG, DM & 73 & 1 & OD & 0.51 & -1.5 & 2.4 \\
\hline 10. H.A. & POAG, DM & 60 & 0.8 & OS & 0.63 & -6.46 & 7.24 \\
\hline 11. G.S. & NTG, DM & 63 & 1 & OD & 0.72 & -5.4 & 3.45 \\
\hline 12. G.C. & POAG, DM & 60 & 0.9 & OS & 0.68 & -0.98 & 2.75 \\
\hline Average & & $60.54 \pm$ & $0.90 \pm$ & & $0.69 \pm$ & $-2.39 \pm$ & $5.56 \pm$ \\
+ S. D. & & 5.45 & 0.41 & & 0.05 & 0.10 & 2.44 \\
\hline
\end{tabular}

Table II: Patients with $\mathrm{OH}$ and diabetes mellitus (group 2), clinical characteristics

\begin{tabular}{|c|c|c|c|c|c|c|c|}
\hline patient & diagnostic & age & VA & eye & C/D & MD & PSD \\
\hline 1. B.M. & OH, DM & 54 & 1 & OD & 0.73 & 0.11 & 1.22 \\
\hline 2. S.E. & OH, DM & 62 & 1 & OD & 0.61 & 0.2 & 1.2 \\
\hline 3. F.O. & OH, DM & 65 & 0.8 & OS & 0.57 & 0.05 & 1.23 \\
\hline 4. B.G. & OH, DM & 65 & 1 & OD & 0.71 & -1.5 & 2.2 \\
\hline 5. G.V. & OH, DM & 65 & 1 & OD & 0.41 & -3.25 & 3.2 \\
\hline $\begin{array}{l}\text { Averag } \\
\text { e } \pm \text { S.D. }\end{array}$ & --- & $62.2 \pm$ & $0.76 \pm$ & & $0.59 \pm$ & $-0.8 \pm$ & $1.81 \pm$ \\
\end{tabular}

The average thickness of the RNFL in the glaucoma and diabetes group was compared with the other two groups, on sectors and it could be noticed a slight reduction of the thickness, especially in the upper and lower sectors, but without statistical significance $(\mathrm{p}=0.238)$ (Table III, Figure 3). There were no statistically significant differences between the intraocular hypertension group and the normal subjects $(p=0.529)$. Regarding the average of the excavation (C/D), there were no significant differences between the three study groups $(\mathrm{p}=0.800)$ (Figure 4). There were statistically significant differences regarding the average RNFL, C/D, between the glaucoma group versus the normal group and the intraocular hypertension group versus the normal group $(\mathrm{p}<0.001)$. 
Table III

Distribution of the modifications of the nerve fibers on sectors in the three groups.

\begin{tabular}{|c|c|c|c|}
\hline Specification & POAG, DM & OH, DM & NORMAL \\
\hline $\begin{array}{c}\text { OCT AVERAGE } \\
\text { SNFR }\end{array}$ & $87.25 \mu \mathrm{m} \pm 11.15$ & $97.8 \mu \mathrm{m} \pm 12.59$ & $90.44 \mu \mathrm{m} \pm 11.46$ \\
\hline SNFR SUP & $109.08 \mu \mathrm{m} \pm 22.15$ & $121 \mu \mathrm{m} \pm 16.35$ & $110.44 \mu \mathrm{m} \pm 14.94$ \\
\hline SNFR INF & $108.25 \mu \mathrm{m} \pm 13.90$ & $121.8 \mu \mathrm{m} \pm 19.47$ & $121.44 \mu \mathrm{m} \pm 24.97$ \\
\hline SNFR NAZAL & $63.75 \mu \mathrm{m} \pm 13.84$ & $68.65 \mu \mathrm{m} \pm 8.90$ & $64.88 \mu \mathrm{m} \pm 6.19$ \\
\hline SNFR TEMPORAL & $65.08 \mu \mathrm{m} \pm 13.58$ & $71.66 \mu \mathrm{m} \pm 18.63$ & $65.55 \mu \mathrm{m} \pm 11.56$ \\
\hline Total & $\mathrm{N}=12$ & $\mathrm{~N}=5$ & $\mathrm{~N}=8$ \\
\hline
\end{tabular}

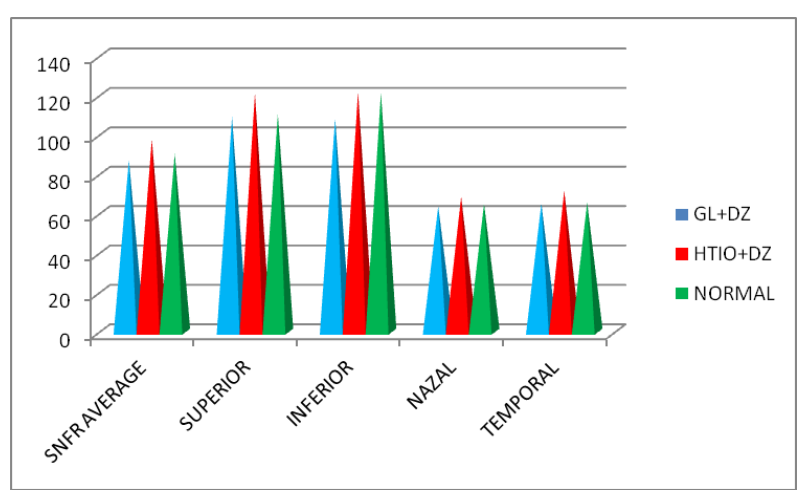

Figure 3. Distribution of the RNFL in the three groups. GL+DM (primary open angle glaucoma and diabetes mellitus), $\mathrm{OH}+\mathrm{DM}$ (intraocular hypertension and diabetes mellitus).

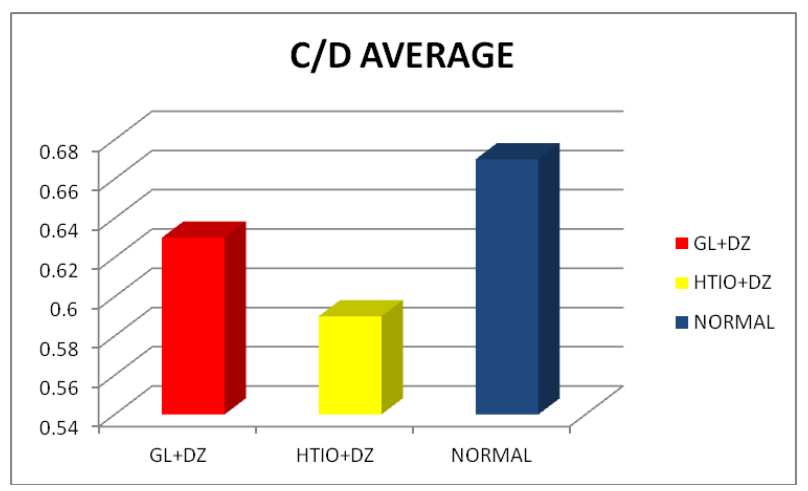

Figure 4. Distribution of the average C/D in the three study GL+DM (primary open angle glaucoma and diabetes mellitus), $\mathrm{OH}+\mathrm{DM}$ (intraocular hypertension and diabetes mellitus).

\section{DISCUSSIONS}

The perimetry deficits in glaucoma occurred when at least $30 \%$ of the ganglion cells were destroyed. Therefore, the early diagnosis of glaucoma was based on the identification of the structural modifications. They must be identified, documented and analyzed in due time, being also important in the monitoring of the progressive optical neuropathy related to glaucoma [2]. The most important quantitative parameters were the total thickness, the thickness in the upper and lower sectors. More studies showed the importance of the OCT evaluation in the early diagnosis of glaucoma [5,6,7,8]. Sahli and co-workers [6], in a comparative study that included 40 glaucoma patients, 55 intraocular hypertension patients and 40 normal subjects, following the evaluation of the retinal nerve fiber layer using OCT, found that the global thickness and on the sectors of the retinal nerve fiber layers was significantly lower as compared to those patients with intraocular hypertension and normal subjects. He also found that there was a positive correlation between the thickness of the RNFL and the visual field results. Huang, in a study conducted on 220 patients[5], found that the evaluation of the thickness of the RNFL was an important parameter in the diagnosis of the pre-perimetric glaucoma. The simultaneous evaluation of the optic disc morphology, the peripapillary-RNFL, the macular-RNFL had an important role in the accuracy of the glaucoma diagnosis. Li and co-workers [7], in a comparative study that included glaucoma 
patients versus normal subjects showed that the best OCT parameters useful in the differentiation of the patients with early glaucoma from the normal subjects were the average and on dials thickness of the RNFL.

In our study, similar to the studies from the specialized literature, the average thickness of the RNFL of the glaucoma patients was smaller than that of the patients with IOHT and the normal subjects, especially in the upper and the lower sector. There were no statistically significant differences between the IOHT group and the normal subjects. There were statistically significant differences regarding the RNFL average, C/D, between the glaucoma group versus the normal subjects and the intraocular hypertension group versus the normal subjects $(\mathrm{p}<0.001)$.

\section{CONCLUSIONS AND FINAL REMARKS}

There were no statistically significant differences between the intraocular hypertension group and the healthy subjects regarding the average thickness of the RNFL. There was a decrease of the thickness of the RNFL for the glaucoma patients and diabetes as compared to the other two groups, but without statistical importance. There were statistically significant differences and a negative correlation in the glaucoma group and the ocular hypertension regarding the MD, PSD and the thickness of the RNFL. Big prospective studies on extended lots of patients were necessary.

\section{ACKNOWLEDGEMENTS}

The authors are very much gratefully acknowledge to the Department of Ophthalmology, Faculty of Medicine, University of Medicine, "Grigore T. Popa" Ias, Romania to provide the data and some most important information related to this experimental work. And in last but not least a lot of thanks to the Physics Department, Faculty of Science, University of Tabuk, Saudi Arabia to complete the present article [9-12].

\section{REFERENCES}

[1] EGS-European Glaucoma Society (author), Terminology and Guidelines for Glaucoma. European Glaucoma Society. $4^{\text {th }}$ ed. 2014.

[2] D. Chiseliță, Glaucomul primitiv cu unghi deschis - gânduri și sinteze practice. Iași: Editura „Gr.T.Popa”, 2014.

[3] D. Huang, E. A. Swanson, C.P. Lin, J.S. Schuman, W. G. Stinson, . Optical coherence tomography, . Science (NCBI, Vol. 254 (5035), 1991, 1178-1181.

[4] N. Anand, The role of ocular coherence tomography in Glaucoma diagnosis and management. Pakistan Journal of Ophthalmology, Vol. 30(1),2014, 1-3.

[5] J.Y. Huang, M. Pekmezci, N. Mesiwala, A. Kao, S. Lin S, Diagnostic power of optic disc morphology, peripapillary retinal nerve fiber layer thickness, and macular inner retinal layer thickness in glaucoma diagnosis with fourier-domain optical coherence tomography, Journal of Glaucoma, Vol. 20 (2), 2011, 87-94.

[6] Esra Şahli, Oya Tekeli, Evaluation of retinal nerve fiber layer thickness with spectral domain oct in primary open angle Glaucoma and ocular hypertension, Journal of Clinical \& Experimental Ophthalmology Vol. 3(8), 2012, 247(1-5).

[7] Li S, Wang X, Li S, Wu G, Wang N., Evaluation of optic nerve head and retinal nerve fiber layer in early and advance glaucoma using frequency domain optical coherence tomography, Graefes Arch. Clin. Exp. Ophthalmol, Vol.248(3), 2010, 429-434.

[8] K. Nouri-Mahdavi, K. Nikkhou, D.C. Hoffman, S.K. Law, J. Caprioli, Detection of early glaucoma with optical coherence tomography (Stratus OCT), Journal of Glaucoma, Vol. 17(3), 2008, 183-188.

[9] Vesna K. Hristova, Julijana Tomovska, Georgi Bonev, Stanimir Dimitrov, M. Ayaz Ahmad, Study of seasonal dynamics of blood metabolic profile and milk urea nitrogen (MUN) level of cows with reproductive disorders" Asian Academic Research Journal of Multidisciplinary (AARJMD), Vol 1 (22),, 2014, 246-260.

[10] Alveera Khan, M. Ayaz Ahmad, Shirish Joshi, A systematic study for electrical properties of chemically treated coir fiber reinforced epoxy composites with ANN model, Int. J. of Sci. and Research (IJSR), Vol. 4(1), 2014, 410-414.

[11] Vesna K. Hristova, M. Ayaz Ahmad, Julijana Tomovska, Biljana Trajkovska, Georgi Bonev, Assessment of raw milk quality by neural networking (N-N) model in Macedonian dairy farms, Int. J. of Ethics in Engn. \& Mang. Educ. (IJEEE), Vol. 1 (10), 2014, 58-61.

[12] M. Ayaz Ahmad, Mir Hashim Rasool, N. Ameer Ahmad, Shafiq Ahmad, Neural networking (N-N) model in relativistic heavy ion collisions, Proceedings International Nuclear Physics Symposium (2013), Vol. 58, 762-763. 